\title{
Visual-spatial training efficacy in children affected by migraine without aura: a multicenter study
}

\author{
This article was published in the following Dove Press journal: \\ Neuropsychiatric Disease and Treatment \\ 27 January 2017 \\ Number of times this article has been viewed
}

\author{
Francesco Precenzano ${ }^{1, *}$ \\ Maria Ruberto ${ }^{2, *}$ \\ Lucia Parisi ${ }^{3}$ \\ Margherita Salerno ${ }^{4}$ \\ Agata Maltese ${ }^{3}$ \\ Beatrice Gallai ${ }^{5}$ \\ Rosa Marotta ${ }^{6}$ \\ Serena Marianna Lavano ${ }^{7}$ \\ Francesco Lavano ${ }^{6}$ \\ Michele Roccella ${ }^{3}$ \\ 'Clinic of Child and Adolescent \\ Neuropsychiatry, Headache Center \\ for Children and Adolescent. \\ Department of Mental and Physical \\ Health and Preventive Medicine, \\ Università degli Studi della Campania \\ Luigi Vanvitelli, ${ }^{2}$ Department \\ of Medical-Surgical and Dental \\ Specialties, Università degli Studi \\ della Campania Luigi Vanvitelli, \\ Naples, ${ }^{3}$ Department of Psychological, \\ Pedagogical and Educational Sciences, \\ ${ }^{4}$ Sciences for Mother and Child \\ Health Promotion, University of \\ Palermo, Palermo, ${ }^{5}$ Department of \\ Surgical and Biomedical Sciences, \\ University of Perugia, Perugia, \\ ${ }^{6}$ Department of Medical and Surgery \\ Sciences, ${ }^{7}$ Department of Health \\ Sciences, University “Magna Graecia”, \\ Catanzaro, Italy \\ *These authors contributed equally \\ to this work
}

Correspondence: Francesco Precenzano Clinic of Child and Adolescent Neuropsychiatry, Headache Center for Children and Adolescent, Department of Mental and Physical Health and Preventive Medicine, Università degli Studi della Campania Luigi Vanvitelli, Naples, Italy

$\mathrm{Tel}+398 \mathrm{I} 5666988$

Fax +39815666694

Email francesco.precenzano@unina2.it

\begin{abstract}
Routinely in the clinical practice, children affected by migraine without aura (MwA) tend to exhibit severe and persistent difficulties within cognitive processes such as attention, memory, and visual-motor integration (VMI) skills. The aim of this study was to assess the visual-spatial and visual-motor abilities among a sample of children with MwA and the effects of a specific computerized training. The study population was composed of 84 patients affected by MwA (39 girls and 45 boys; mean age: $8.91 \pm 2.46$ years), and they were randomly divided into two groups (group A and group B) comparable for age $(P=0.581)$, gender $(P=0.826)$, socioeconomic status (SES), migraine frequency $(P=0.415)$, and intensity $(P=0.323)$. At baseline (T0), the two groups were comparable for movement assessment battery for children (M-ABC) and VMI performances. After 6 months of treatment (T1), group A showed lower scores in the dexterity item of $\mathrm{M}-\mathrm{ABC}$ test $(P<0.001)$ and higher scores in $\mathrm{M}-\mathrm{ABC}$ global performance centile $(P<0.001)$ and total $(P<0.001)$, visual $(P=0.017)$, and motor $(P<0.001)$ tasks of VMI test than group B. Moreover, at T1, group A showed higher scores in total $(P<0.001)$ and motor $(P<0.001)$ tasks of VMI test and in M-ABC global performance centile $(P<0.001)$ and lower scores in the dexterity item of M-ABC test $(P<0.001)$ than at T0. Group B showed, at T1, performances comparable to $\mathrm{T} 0$ for all evaluations. As reported by recent studies about alteration MwA among children in motor abilities, our study confirmed these difficulties and the efficacy of a specific software training, suggesting a new rehabilitative proposal in childhood.
\end{abstract}

Keywords: migraine without aura, visual-motor integration, visual-motor training skills

\section{Introduction}

According to the International Classification of Headache Disorders, third version (ICHD-3), migraine has been defined as a common disabling primary headache disorder as ranked in the Global Burden of Disease Survey 2010 that identified migraine as one of the most prevalent disorders and the seventh highest specific cause of disability worldwide. ${ }^{1,2}$ Particularly, in pediatric age, migraine without aura (MwA) impacts different life aspects such as cognition, sleep habits, metabolic regulation, sociality, parenting, and attachment styles ${ }^{3-19}$ and seems to be more frequent than estimated, with a 1 -year prevalence of $9.1 \%$ in children ${ }^{20}$ and up to $36 \%$ among adolescents. ${ }^{21}$

Recent studies have shown that in addition to the difficulties in performing daily activities, children and adolescents with migraine tend to present typical cognitive and behavioral changes during the attack and various levels of impairment between the attacks. ${ }^{22}$ Typically, a significant reduction in information speed processing has been reported in migraine sufferers that has also impaired visual-spatial memory, in verbal performances and attentive skills. ${ }^{23}$ These difficulties seem to be related to deficits in strategic and organizational aspects of learning processing. On the other hand, migraineurs have also found differences in visual cortical processing compared 
to healthy subjects. ${ }^{24}$ In particular, the ability to identify the overall direction of motion seems impaired, pinpointing that movement problems frequent in children and adolescents with MwA appear to be purely perceptual origin. ${ }^{25}$ Moreover, Esposito et $\mathrm{a}^{11}$ reported the association between poor motor coordination and MwA in children suggesting a role of impairment in perceptual organization abilities of children with MwA as the cause for motor coordination and visualmotor integration (VMI) impairment of these subjects. ${ }^{26}$ In this context, we previously proposed a new rehabilitation approach to these conditions in children affected by MwA, ${ }^{27}$ suggesting a positive role of exergames in motor coordination improvement, although only partial improvement in VMI abilities has been obtained.

The aim of the this study was to assess the effect of a specific software for visual-spatial training on VMI abilities in children affected by MwA.

\section{Methods}

The study population was composed of 84 patients affected by MwA (39 girls and 45 boys; mean age: $8.91 \pm 2.46$ years), consecutively referred to the tertiary level Center for Childhood Headache of the Department of Child and Adolescent Neuropsychiatry, Università degli Studi della Campania Luigi Vanvitelli, University of Palermo, University of Perugia, and University of Catanzaro.

MwA diagnosis was performed according to the ICHD-3 criteria. $^{1}$

Exclusion criteria were the following: prematurity and/or lower gestation (birth weight), ${ }^{28,29}$ mental retardation (IQ $<70$ ), borderline intellectual functioning $(71<\mathrm{IQ}<85),{ }^{30,31}$ genetic syndromes (eg, down syndrome, Prader-Willi syndrome, and fragile X syndrome), ${ }^{32-34}$ hypothyroidism, ${ }^{35}$ psychiatric disorders (schizophrenia, mood disorders, and attention-deficit hyperactivity disorder [ADHD] $),{ }^{36}$ movement disorders, neuromuscular disorders, epilepsy, ${ }^{37-39}$ and obesity. ${ }^{40-42}$

All the subjects were Caucasian. As previously reported, ${ }^{43}$ a semi-structured interview (Hollingshead Four-Factor Index of Social Status $)^{44}$ was conducted to collect information from the parents to assess the socioeconomic status (SES). The whole population underwent a clinical evaluation to assess the VMI and motor coordination skills at baseline (T0), and a headache diary was kept to collect the frequency of attacks and estimate migraine intensity according to a 5-point visual analog scale (VAS) evaluation.

For migraine attacks, all the enrolled children were treated with a preventive therapy, such as Ginkgolide-B complex, as previously reported ${ }^{45-47}$ during the entire study period.
After T0 evaluation, the study group was randomly divided into two groups (group A and group B) composed of 42 children in each group. Group A underwent a specific training course for 6 months using the software "Allenare le abilità visuo-spaziali”, commonly used for coaching visual-spatial skills in pediatric age. ${ }^{48}$ The training software was present in each participant's study center, and all the recruited children followed the same protocol training, for three times per week and for a duration of 2 hours for each session. The patient treatment compliance for $100 \%$ and no adverse effects have been reported. For group B, only migraine preventive therapy was administered.

After 6 months of treatment (T1), the two groups underwent a clinical evaluation to compare the VMI and motor coordination skills.

Written informed consent was obtained from the parents of all children. Departmental ethics committees at the University of Palermo, Perugia and Catanzaro approved the study protocol (EudraCT number: 2015-001161-36).

\section{Evaluation of motor coordination skills}

The impairment of motor coordination performance related to age expectations was determined using the movement assessment battery for children (M-ABC) test. This test is frequently used in both clinical and research settings to assess children for motor coordination impairment and has high reliability and validity ${ }^{49}$ In fact, it assesses fine and gross motor skills using three manual dexterity tasks, two ball skills tasks, and three balance tasks, each of which is scored on a 5-point scale. The raw score of each item is then converted to a score scale ranging from 0 to 5 . The higher score indicates a less-than-adequate performance. Consequently, 0 reflects a complete success by the candidate on the task examined, while 5 reflects a failure in the execution of the task; in fact, failed (F), inappropriate $(\mathrm{I})$, or refused (R) performances are transformed into 5. The sum of the eight scores of items corresponds to the total score of disability, ranging between 0 and 40 , where a lower score is a result of implementing the best move. The content of the items differs depending on the age of the child examined, with increasing difficulty according to age, so that the battery is made up of four different types of activities considered to be made in relation to age (4-6 years, 7-8 years, 9-10 years, and 11-12 years). Each subject was assessed individually in $\sim 20-40 \mathrm{~min} .{ }^{49}$ The total impairment score was calculated from these individual tasks and used to generate a centile score compared to the standardization sample.

\section{Evaluation of VMI skills}

The fine motor coordination and the VMI were assessed by the Beery VMI task, ${ }^{50}$ a paper-and-pencil test, where 
children had to imitate or copy up to 27 geometric forms with increasing complexity using paper and pencil. Copying errors were marked if they reflected problems in fine motor coordination, rather than a pure visuospatial problem. The task is specifically designed for children and takes $\sim 10 \mathrm{~min}$. The Beery VMI scores were standardized for age and gender using normative data for the Italian general population. ${ }^{50}$ The centile scores were used for diagnosing the visual-motor abnormalities in our sample, and a value of $\leq 5$ was considered for VMI impairment.

\section{Software "Allenare le abilità visuo-spaziali”}

For the treatment and strengthening of visual-spatial skills and improving the nonverbal activities, particularly useful in the context of learning, this software offers in the form of classic games and tests a series of exercises that stimulate visual perception, such as rotation of figures, the playback of a track, the reconstruction of an image or a photo, the orientation of visual configurations, and the spatial organization. These exercises are structured in three levels of difficulty and focus on the natural interest of the kids toward games and video games.

One can check the results of each player in each activity and set the options to adapt the program to the specific needs of navigation through a section dedicated to software management. $^{48}$

\section{Statistical analysis}

For comparison between the two groups (group A and group B), paired $t$-test and, where appropriate, the Chi-square test were applied at T0 and T1.

Repeated-measures ANOVA was performed to compare the training effects within each group between $\mathrm{T} 0$ (baseline) and after 6 months of training (T1). $P$-values $<0.05$ were considered statistically significant.

For statistical analysis, the software "STATISTICA" (data analysis software system, version 6; StatSoft, Inc., 2001) was used.

\section{Results}

The study population was composed of 84 patients affected by MwA (39 girls and 45 boys; mean age: $8.91 \pm 2.46$ years), and they were randomly divided into two groups (group A and group B). In the two groups, there were no statistical differences in age $(P=0.581)$, gender $(P=0.826)$, SES (Table 1$)$, migraine frequency $(P=0.415)$, and intensity $(P=0.323$; Table 1$)$.

At T0, in the two groups, no statistical differences were found for M-ABC and VMI performances (Table 2).
Table I Comparison between the two study groups for demographic and migraine characteristics at TO

\begin{tabular}{llll}
\hline Characteristics & $\begin{array}{l}\text { Group A } \\
(\mathbf{N}=42)\end{array}$ & $\begin{array}{l}\text { Group B } \\
\mathbf{( N = 4 2 )}\end{array}$ & P-value \\
\hline Age (years) & $8.35 \pm \mathrm{I} .67$ & $8.13 \pm \mathrm{I} .96$ & $0.58 \mathrm{I}$ \\
Gender & $24 \mathrm{~B} / \mathrm{I} 8 \mathrm{G}$ & $22 \mathrm{M} / 20 \mathrm{~F}$ & 0.826 \\
Migraine frequency (monthly) & $10.37 \pm \mathrm{I} .28$ & $10.65 \pm \mathrm{I} .8 \mathrm{I}$ & $0.4 \mathrm{I}$ \\
Migraine intensity (VAS scale) & $3.92 \pm \mathrm{I} .04$ & $3.68 \pm \mathrm{I} .17$ & 0.323 \\
SES: high & $9(2 \mathrm{I} .43 \%)$ & $\mathrm{II}(26.19 \%)$ & 0.798 \\
SES: medium & $27(64.28 \%)$ & $23(54.76 \%)$ & 0.505 \\
SES: low & $6(14.29 \%)$ & $8(19.05 \%)$ & 0.770 \\
\hline
\end{tabular}

Notes: T0, baseline. Data presented as mean \pm standard deviation unless otherwise indicated.

Abbreviations: B, boys; G, girls; SES, socioeconomic status.

At T1, group A showed lower scores in the dexterity item of $\mathrm{M}-\mathrm{ABC}$ test $(P<0.001)$ and higher scores in $\mathrm{M}-\mathrm{ABC}$ global performance centile $(P<0.001)$ and total $(P<0.001)$, visual $(P=0.017)$, and motor $(P<0.001)$ tasks of VMI test than group B (Table 3 ).

Moreover, at T1, group A showed higher scores in total $(P<0.001)$ and motor $(P<0.001)$ tasks of VMI test and in $\mathrm{M}-\mathrm{ABC}$ global performance centile $(P<0.001)$ and lower scores in the dexterity item of M-ABC test $(P<0.001)$ than T0 (Table 4).

Group B showed, at T1, performances that were not statistically different with respect to T0 for all evaluations (Table 5).

\section{Discussion}

Despite considerable progress in the study of the pathophysiology of headache, the effects of migraine in daily life are not yet fully understood, especially in the developmental age. Reports on cognitive functioning and visual-spatial abilities of children with migraine during the interictal phase are scarce and conflicting.

D'Andrea et $\mathrm{al}^{51}$ evaluated 20 children with MwA aged 7-11 years, not showing impairment in cognitive functioning,

Table 2 Comparison between the two study groups for $\mathrm{VMI}$ and $\mathrm{M}-\mathrm{ABC}$ evaluations at TO

\begin{tabular}{|c|c|c|c|}
\hline $\begin{array}{l}\text { M-ABC and } \\
\text { VMI scores }\end{array}$ & $\begin{array}{l}\text { Group A } \\
(N=42)\end{array}$ & $\begin{array}{l}\text { Group B } \\
(\mathrm{N}=42)\end{array}$ & $P$-value \\
\hline Dexterity & $12.36 \pm 9.44$ & $12.05 \pm 10.23$ & 0.886 \\
\hline Ball skills & $3.91 \pm 1.27$ & $4.03 \pm 2.05$ & 0.748 \\
\hline Balance & $13.85 \pm 10.72$ & $|4.01 \pm 1| .06$ & 0.946 \\
\hline M-ABC total score & $30.91 \pm 22.56$ & $31.09 \pm 22.73$ & 0.971 \\
\hline M-ABC centile & $8.87 \pm 3.09$ & $9.02 \pm 3.16$ & 0.826 \\
\hline VMI total score & $29.51 \pm 13.78$ & $29.66 \pm 14.02$ & 0.961 \\
\hline VMI: visual task & $49.07 \pm 25.89$ & $48.93 \pm 26.05$ & 0.980 \\
\hline VMI: motor task & II.14士7.38 & $11.76 \pm 7.19$ & 0.698 \\
\hline
\end{tabular}

Notes: T0, baseline. Data presented as mean \pm standard deviation.

Abbreviations: $M-A B C$, movement assessment battery for children; VMI, visualmotor integration. 
Table 3 Comparison between the two study groups for VMI and $\mathrm{M}-\mathrm{ABC}$ evaluations at $\mathrm{TI}$

\begin{tabular}{llll}
\hline & $\begin{array}{l}\text { Group A } \\
(\mathbf{N}=\mathbf{4 2})\end{array}$ & $\begin{array}{l}\text { Group B } \\
\mathbf{( N = 4 2 )}\end{array}$ & P-value \\
\hline Dexterity & $5.23 \pm 1.18$ & $1 \mathrm{I} .96 \pm 9.37$ & $<0.00 \mathrm{I}$ \\
Ball skills & $3.92 \pm 2.49$ & $4.17 \pm 2.28$ & 0.633 \\
Balance & $13.97 \pm 10.92$ & $13.64 \pm 10.46$ & 0.888 \\
M-ABC total score & $25.31 \pm 18.26$ & $33.08 \pm 18.09$ & 0.054 \\
M-ABC centile & $13.08 \pm 3.67$ & $8.87 \pm 3.4 \mid$ & $<0.00 \mathrm{I}$ \\
VMI total score & $45.17 \pm 16.96$ & $28.73 \pm \mid 3.26$ & $<0.001$ \\
VMI: visual task & $57.26 \pm 18.73$ & $45.32 \pm 25.58$ & 0.017 \\
VMI: motor task & $20.45 \pm 10.87$ & $12.03 \pm 7.95$ & $<0.00 \mathrm{I}$ \\
\hline
\end{tabular}

Notes: TI, after 6 months of treatment. Data presented as mean \pm standard deviation.

Abbreviations: M-ABC, movement assessment battery for children; VMI, visualmotor integration.

except for performances that were significantly compromised in the short- and long-term memory tasks.

Moreover, Calandre et al $^{52}$ suggested that visuomotor processing speed may be considered the first sign of neuropsychological dysfunction in adults with migraine.

Conversely, Esposito et $\mathrm{al}^{26}$ conducted the first study on the association between poor motor coordination and MwA in children, using standardized tools, highlighting the higher prevalence of developmental coordination disorder and VMI impairment than controls.

Again, the same study reported a lower performance at the M-ABC test and in motor coordination task of VMI test in children with migraine than controls. In this light, our findings seem to show a positive impact on visualspatial abilities of children with MwA, highlighting the role of overall management of unusual comorbidities of migraine.

On the other hand, as reported by Smits-Engelsman et $a l,{ }^{53}$ repeating the same task in a controlled way using

Table 4 Comparison between VMI and M-ABC evaluations at T0 and TI for group A

\begin{tabular}{llll}
\hline & T0 & TI & $P$-value \\
\hline Dexterity & $12.36 \pm 9.44$ & $5.23 \pm I .18$ & $<0.00 \mathrm{I}$ \\
Ball skills & $3.91 \pm 1.27$ & $3.92 \pm 2.49$ & 0.982 \\
Balance & $13.85 \pm 10.72$ & $13.97 \pm 10.92$ & 0.960 \\
M-ABC total score & $30.91 \pm 22.56$ & $25.31 \pm 18.26$ & 0.215 \\
M-ABC centile & $8.87 \pm 3.09$ & $13.08 \pm 3.67$ & $<0.00 \mathrm{I}$ \\
VMI total score & $29.5 \mathrm{I} \pm 13.78$ & $45.17 \pm 16.96$ & $<0.00 \mathrm{I}$ \\
VMI: visual task & $49.07 \pm 25.89$ & $57.26 \pm 18.73$ & $0.10 \mathrm{I}$ \\
VMI: motor task & $1 \mathrm{I} .14 \pm 7.38$ & $20.45 \pm 10.87$ & $<0.00 \mathrm{I}$ \\
\hline
\end{tabular}

Notes: T0, baseline; TI, after 6 months of treatment. Data presented as mean \pm standard deviation.

Abbreviations: M-ABC, movement assessment battery for children; VMI, visualmotor integration.
Table 5 Comparison between VMI and M-ABC evaluations at T0 and TI for group B

\begin{tabular}{llll}
\hline & T0 & TI & $P$-value \\
\hline Dexterity & $12.05 \pm 10.23$ & $1 \mathrm{I} .96 \pm 9.37$ & 0.967 \\
Ball skills & $4.03 \pm 2.05$ & $4.17 \pm 2.28$ & 0.768 \\
Balance & $14.0 I \pm I I .06$ & $13.64 \pm 10.46$ & 0.875 \\
M-ABC total score & $31.09 \pm 22.73$ & $33.08 \pm 18.09$ & 0.658 \\
M-ABC centile & $9.02 \pm 3.16$ & $8.87 \pm 3.4 \mid$ & 0.835 \\
VMI total score & $29.66 \pm 14.02$ & $28.73 \pm 13.26$ & 0.756 \\
VMI: visual task & $48.93 \pm 26.05$ & $45.32 \pm 25.58$ & 0.523 \\
VMI: motor task & $11.76 \pm 7.19$ & $12.03 \pm 7.95$ & $0.87 \mid$ \\
\hline
\end{tabular}

Notes: T0, baseline; TI, after 6 months of treatment. Data presented as mean \pm standard deviation.

Abbreviations: M-ABC, movement assessment battery for children; VMI, visualmotor integration.

a standardized exergaming learning session seems to be a good way to examine motor learning skills. In this light, using virtual environments can be identified as beneficial to the efficiency and outcome of rehabilitative program in children with disabling medical conditions or lower level of physical fitness. ${ }^{27}$ Moreover, considering that children with migraine are identified as less inclined to engaging in physical activities, ${ }^{27}$ stimulating them through exergaming and/ or software training (active computer games), which they enjoy, may be useful to promote their motor skills. On the other hand, many reports showed specific links between neuropsychological impairment and neuroimaging findings in migraine subjects in both adults and children. Schmitz et al ${ }^{54}$ demonstrated in adults with migraine a correlation between executive function impairment and density deficit in frontal and parietal gray matter. Moreover, Rocca et $\mathrm{al}^{55}$ showed in children affected by migraine atrophy in several gray matter regions of temporal and frontal lobes, including the cingulum, which are involved in executive functioning.

Alternatively, the executive functioning impairment and abnormalities in frontal regions may induce a reduction in perceptual organization abilities in children with MwA. Such changes may also affect motor coordination and visualmotor skills that seem to explain the results of this study with respect to the high prevalence of borderline motor skills in children with MwA.

\section{Conclusion}

The current study demonstrates the effectiveness of visuospatial training with specific software for the enhancement of visuospatial abilities in children with MwA, emphasizing the importance of precocious evaluation and management of all aspects of migraine comorbidities. 


\section{Acknowledgment}

We thank Giovanni Messina, MD, PhD, Senior Researcher, working at Foggia University and Naples University for his intellectual contribution to the manuscript.

\section{Disclosure}

The authors report no conflicts of interest in this work.

\section{References}

1. Headache Classification Committee of the International Headache Society (IHS). The International Classification of Headache Disorders. Vol. 33. 3rd ed. Cephalalgia; 2013:629-808.

2. Charlson FJ, Baxter AJ, Dua T, Degenhardt L, Whiteford HA, Vos T. Excess mortality from mental, neurological, and substance use disorders in the global burden of disease study 2010. In: Patel V, Chisholm D, Dua T, Laxminarayan R, Medina-Mora ME, editors. Mental, Neurological, and Substance Use Disorders: Disease Control Priorities. Vol 4. 3rd ed. Washington, DC: The International Bank for Reconstruction and Development/The World Bank; 2016:Chapter 3.

3. Balottin U, Chiappedi M, Rossi M, Termine C, Nappi G. Childhood and adolescent migraine: a neuropsychiatric disorder? Med Hypotheses. 2011;76(6):778-781.

4. Piazza F, Chiappedi M, Maffioletti E, Galli F, Balottin U. Medication overuse headache in school-aged children: more common than expected? Headache. 2012;52(10):1506-1510.

5. De Carlo D, Dal Zotto L, Perissinotto E, et al. Osmophobia in migraine classification: a multicentre study in juvenile patients. Cephalalgia. 2010; 30(12):1486-1494

6. Balottin U, Fusar Poli P, Termine C, Molteni S, Galli F. Psychopathological symptoms in child and adolescent migraine and tension-type headache: a meta-analysis. Cephalalgia. 2013;33(2):112-122.

7. Esposito M, Parisi P, Miano S, Carotenuto M. Migraine and periodic limb movement disorders in sleep in children: a preliminary case-control study. J Headache Pain. 2013;14:57.

8. Carotenuto M, Esposito M, Pascotto A. Migraine and enuresis in children: an unusual correlation? Med Hypotheses. 2010;75(1):120-122.

9. Carotenuto M, Esposito M, Precenzano F, Castaldo L, Roccella M. Cosleeping in childhood migraine. Minerva Pediatr. 2011;63(2): 105-109.

10. Verrotti A, Agostinelli S, D'Egidio C, et al. Impact of a weight loss program on migraine in obese adolescents. Eur J Neurol. 2013;20(2): 394-397.

11. Esposito M, Pascotto A, Gallai B, et al. Can headache impair intellectual abilities in children? An observational study. Neuropsychiatr Dis Treat 2012;8:509-513.

12. Esposito M, Gallai B, Parisi L, et al. Maternal stress and childhood migraine: a new perspective on management. Neuropsychiatr Dis Treat. 2013;9:351-355.

13. Esposito M, Gallai B, Parisi L, et al. Self-concept evaluation and migraine without aura in childhood. Neuropsychiatr Dis Treat. 2013;9: 1061-1066.

14. Esposito M, Antinolfi L, Gallai B, et al. Executive dysfunction in children affected by obstructive sleep apnea syndrome: an observational study. Neuropsychiatr Dis Treat. 2013;9:1087-1094.

15. Esposito M, Marotta R, Gallai B, et al. Temperamental characteristics in childhood migraine without aura: a multicenter study. Neuropsychiatr Dis Treat. 2013;9:1187-1192.

16. Esposito M, Roccella M, Gallai B, et al. Maternal personality profile of children affected by migraine. Neuropsychiatr Dis Treat. 2013;9: 1351-1358.

17. Esposito M, Parisi L, Gallai B, et al. Attachment styles in children affected by migraine without aura. Neuropsychiatr Dis Treat. 2013;9: 1513-1519.
18. Verrotti A, Carotenuto M, Altieri L, et al. Migraine and obesity: metabolic parameters and response to a weight loss programme. Pediatr Obes. 2015;10(3):220-225.

19. Messina A, De Fusco C, Monda V, et al. Role of the orexin system on the hypothalamus-pituitary-thyroid axis. Front Neural Circuits. 2016; 10:66.

20. Wöber-Bingöl C. Epidemiology of migraine and headache in children and adolescents. Curr Pain Headache Rep. 2013;17(6):341.

21. Krogh AB, Larsson B, Linde M. Prevalence and disability of headache among Norwegian adolescents: a cross-sectional school-based study Cephalalgia. 2015;35(13):1181-1191.

22. Balottin U, Termine C. Recommendations for the management of migraine in paediatric patients. Expert Opin Pharmacother. 2007;8(6): 731-744.

23. Farmer K, Cady R, Bleiberg J, Reeves D. A pilot study to measure cognitive efficiency during migraine. Headache. 2000;40(8):657-661.

24. Harriott AM, Schwedt TJ. Migraine is associated with altered processing of sensory stimuli. Curr Pain Headache Rep. 2014;18(11):458

25. Lanzi G, Balottin U, Borgatti R, Guderzo M, Scarabello E. Different forms of migraine in childhood and adolescence: notes on personality traits. Headache. 1988;28(9):618-622.

26. Esposito M, Verrotti A, Gimigliano F, et al. Motor coordination impairment and migraine in children: a new comorbidity? Eur J Pediatr. 2012; 171(11):1599-1604.

27. Esposito M, Ruberto M, Gimigliano F, et al. Effectiveness and safety of Nintendo Wii Fit Plus ${ }^{\mathrm{TM}}$ training in children with migraine without aura: a preliminary study. Neuropsychiatr Dis Treat. 2013;9:1803-1810.

28. Guzzetta A, Pizzardi A, Belmonti V, et al. Hand movements at 3 months predict later hemiplegia in term infants with neonatal cerebral infarction. Dev Med Child Neurol. 2010;52(8):767-772.

29. Guzzetta A, D'Acunto MG, Carotenuto M, et al. The effects of preterm infant massage on brain electrical activity. Dev Med Child Neurol. 2011; 53(suppl 4):46-51.

30. Esposito M, Carotenuto M. Intellectual disabilities and power spectra analysis during sleep: a new perspective on borderline intellectual functioning. J Intellect Disabil Res. 2014;58(5):421-429.

31. Esposito M, Carotenuto M. Borderline intellectual functioning and sleep: the role of cyclic alternating pattern. Neurosci Lett. 2010;485(2): 89-93.

32. Morandi A, Bonnefond A, Lobbens S, et al. A girl with incomplete PraderWilli syndrome and negative MS-PCR, found to have mosaic maternal UPD-15 at SNP array. Am J Med Genet A. 2015;167A(11):2720-2726.

33. Verrotti A, Cusmai R, Laino D, et al. Long-term outcome of epilepsy in patients with Prader-Willi syndrome. J Neurol. 2015;262(1):116-123. Erratum in: J Neurol. 2015;262(1):124-125. Carotenuto, Marco [added]; Esposito, Maria [added].

34. Carotenuto M, Esposito M, D'Aniello A. Polysomnographic findings in Rett syndrome: a case-control study. Sleep Breath. 2013;17(1):93-98. Erratum in: Sleep Breath. 2013;17(2):877-878.

35. Pasquali D, Carotenuto M, Leporati P, et al. Maternal hypothyroidism and subsequent neuropsychological outcome of the progeny: a family portrait. Endocrine. 2015;50(3):797-801.

36. Cavanna AE, David K, Bandera V, et al. Health-related quality of life in Gilles de la Tourette syndrome: a decade of research. Behav Neurol. 2013;27(1):83-93.

37. Franzoni E, Matricardi S, Di Pisa V, et al. Refractory absence seizures: an Italian multicenter retrospective study. Eur J Paediatr Neurol. 2015; 19(6):660-664.

38. Carotenuto M, Parisi P, Esposito M, Cortese S, Elia M. Sleep alterations in children with refractory epileptic encephalopathies: a polysomnographic study. Epilepsy Behav. 2014;35:50-53.

39. Bellini B, Arruda M, Cescut A, et al. Headache and comorbidity in children and adolescents. J Headache Pain. 2013;14:79.

40. Carotenuto M, Santoro N, Grandone A, et al. The insulin gene variable number of tandem repeats (INS-VNTR) genotype and sleep disordered breathing in childhood obesity. J Endocrinol Invest. 2009; 32(9):752-755. 
41. Carotenuto M, Bruni O, Santoro N, Del Giudice EM, Perrone L, Pascotto A. Waist circumference predicts the occurrence of sleepdisordered breathing in obese children and adolescents: a questionnairebased study. Sleep Med. 2006;7(4):357-361.

42. Esposito M, Gallai B, Roccella M, et al. Anxiety and depression levels in prepubertal obese children: a case-control study. Neuropsychiatr Dis Treat. 2014;10:1897-1902.

43. Luoni C, Balottin U, Rosana L, Savelli E, Salini S, Termine C. Confrontation naming and reading abilities at primary school: a longitudinal study. Behav Neurol. 2015;2015:981548.

44. Hollingshead AB. Four Factor Index of Social Status. New Haven, CT: Department of Sociology, Yale University; 1975.

45. Carotenuto M, Esposito M. Nutraceuticals safety and efficacy in migraine without aura in a population of children affected by neurofibromatosis type I. Neurol Sci. 2013;34(11):1905-1909.

46. Esposito M, Ruberto M, Pascotto A, Carotenuto M. Nutraceutical preparations in childhood migraine prophylaxis: effects on headache outcomes including disability and behaviour. Neurol Sci. 2012;33(6): 1365-1368.

47. Esposito M, Carotenuto M. Ginkgolide B complex efficacy for brief prophylaxis of migraine in school-aged children: an open-label study. Neurol Sci. 2011;32(1):79-81.
48. Miato L, Andrich S. Allenare le abilità visuo-spaziali. Erickson; 2008.

49. Henderson SE, Sugden DA. Movement Assessment Battery for Children Manual. London: The Psychological Corporation Ltd; 1992.

50. Beery KE, Beery NA. The Beery-Buktenica Developmental Test of Visual-Motor Integration. Vol. 5. Minneapolis, MN: NCS Pearson Inc; 2004

51. D'Andrea G, Nertempi P, Ferro Milone F, et al. Personality and memory in childhood migraine. Cephalagia. 1989;9:25-28.

52. Calandre EP, Bembibre J, Arnedo ML, Beccera D. Cognitive disturbances and regional cerebral blood flow abnormalities in migraine patients: their relationship with the clinical manifestations of the illness. Cephalagia. 2002;22(4):291-302.

53. Smits-Engelsman BC, Jelsma LD, Ferguson GD, Geuze RH. Motor learning: an analysis of 100 trials of a Ski Slalom Game in children with and without developmental coordination disorder. PLoS One. 2015; 10(10): e0140470.

54. Schmitz N, Arkink EB, Mulder M, et al. Frontal lobe structure and executive function in migraine patients. Neurosci Lett. 2008;440(2):92-96.

55. Rocca MA, Messina R, Colombo B, Falini A, Comi G, Filippi M. Structural brain MRI abnormalities in pediatric patients with migraine. J Neurol. 2014;261(2):350-357.

\section{Publish your work in this journal}

Neuropsychiatric Disease and Treatment is an international, peerreviewed journal of clinical therapeutics and pharmacology focusing on concise rapid reporting of clinical or pre-clinical studies on a range of neuropsychiatric and neurological disorders. This journal is indexed on PubMed Central, the 'PsycINFO' database and CAS,

\section{Dovepress}

and is the official journal of The International Neuropsychiatric Association (INA). The manuscript management system is completely online and includes a very quick and fair peer-review system, which is all easy to use. Visit http://www.dovepress.com/testimonials.php to read real quotes from published authors. 